\title{
Algorithm for pre-emptive glycopeptide treatment in patients with haematologic malignancies and an Enterococcus faecium bloodstream infection
}

\author{
Xuewei Zhou ${ }^{1 *}$, Jan P Arends ${ }^{1}$, Lambert FR Span ${ }^{2}$ and Alexander W Friedrich ${ }^{1}$
}

\begin{abstract}
Introduction: Nowadays Enterococcus faecium has become one of the most emerging and challenging nosocomial pathogens. The aim of this study was to determine risk factors in haematology patients who are at risk of an Enterococcus faecium bloodstream infection (BSI) and should be considered for pre-emptive glycopeptide treatment. With these identified risk factors a prediction model can be developed for clinical use.

Methods: Retrospectively clinical and microbiological data in 33 patients with an E. faecium BSI were compared to 66 control patients during a 5-year period at the haematology ward. Multivariate logistic regression was used to explore the independent risk factors and a prediction model was developed to determine the risk of an E. faecium BSI.

Results: E. faecium BSIs were found to be associated with high mortality rates. Independent risk factors for $E$. faecium BSI were colonization with E. faecium 30 days prior to blood culture (OR 5.71; Cl 1.7-18.7), combination of neutropenia and abdominal focus $(4.37 ; 1.4-13.4)$, age $>58$ years $(4.01 ; 1.3-12.5)$, hospital stay prior to blood culture $>14$ days (3.55; 0.98-12.9) and CRP (C-reactive protein) level $>125$ mg/L (4.37; 1.1-10.2).

Conclusion: Using data from this study, risk stratification for the development of an E. faecium BSI in patients with haematological malignancies is possible. Pre-emptive treatment should be considered in those patients who are at high risk. Using a prediction model as designed in this study, antibiotic stewardship in terms of prudent use of glycopeptides can be improved and might be helpful in controlling further spread of VRE (vancomycin resistant enterococci).
\end{abstract}

Keywords: Enterococcus faecium, Haematologic patients, Risk factors, Glycopeptides, Antibiotic stewardship

\section{Introduction}

Enterococcus faecium has become one of the most important, emerging and challenging nosocomial pathogens [1]. It is a difficult to treat pathogen due to intrinsic resistances to cephalosporins, aminoglycosides (low-level resistance), clindamycin and trimethoprimsulfamethoxazole [2]. Moreover, it has the ability to easily acquire virulence or antibiotic resistance genes trough transfer of plasmids, chromosomal exchange or mutation [3].

\footnotetext{
* Correspondence: x.w.zhou@umcg.nl

'Department of Medical Microbiology, University of Groningen, University

Medical Center Groningen, Groningen, The Netherlands

Full list of author information is available at the end of the article
}

Due to the resistance of multiple antibiotics, the treatment of choice in serious E. faecium infections is glycopeptides. However, prudent use of vancomycin is needed as it is associated with an increased risk for VRE infection and colonization [4]. The emergence of VRE has been reported one to two decades ago in the United States [5]; more recently alarming reports are now coming from many countries in Europe [6].

Several studies have pointed out the existence of two subpopulations of E. faecium: commensal/communityassociated (CA) strains and clinical or hospital associated (HA) strains, whereas the latter is also referred as the clonal complex 17 (CC-17) group [7]. These HA/CC-17 strains are associated with ampicillin resistance; the rise

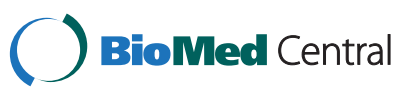


and replacement of E. faecium as the predominant enterococcus species are especially due to these strains [8].

A predominant part of the nosocomial E. faecium bloodstream infections concerns patients with haematologic malignances who are immunocompromised by their severe disease and intensive treatment. Whereas it often is debated whether to treat E. faecium as a real pathogen, several studies have shown high morbidity and mortality rates for enterococcal bacteremia (mortality rates ranging from $25 \%$ to $51 \%$ ), especially in immunocompromised patients [9-11]. Moreover, the mortality rates increases with inappropriate antimicrobial therapy [12].

After coagulase negative staphylococci (CoNS), streptococci and Escherichia coli (E. coli), E. faecium is the most predominant species isolated among blood cultures at the haematology unit of our hospital. Compared to other pathogens such as CoNS, E. coli, Pseudomonas aeruginosa (P. aeruginosa) and streptococci which remained stable or decreased, E. faecium increased for the periods 1998-2006 (3.1\%) and 2007$2010(12.8 \%)$ which is 4.1 times more.

Since patients with haematologic malignancies are highly prone to infection, prophylactic antibiotics are used to prevent and reduce any risk of infection. In our haematology ward penicillin and ciprofloxacin or cotrimoxazol or colistine or tobramycin (orally) are used depending on the resistance pattern of bacteria found in surveillance cultures. In case a haematology patient presents with neutropenic fever or other clinical signs of infection, blood cultures are taken and empirical broadspectrum antibiotic treatment is started, which is piperacillin-tazobactam.

Glycopeptides are not recommended as a standard part of the initial antibiotic regimen for fever and neutropenia. Moreover, as noted earlier, for the further prevention and control of VRE it is necessary to control the use of glycopeptide antibiotics. At this moment, glycopeptides are only added in case of a positive blood culture with $E$. faecium or oxacillin resistant CoNS. However blood culture results and their susceptibilities are only available after one or more days after blood samples are drawn.

Therefore the aim of this study is to identify possible risk factors in those haematology patients who are at high risk of $E$. faecium bloodstream infection in order to develop a prediction model for clinical stringent use. This can be useful in the decision of pre-emptive therapy with glycopeptides together with the initial empirical antibiotic treatment at the moment a blood culture is taken.

\section{Methods}

Study design and population

The University Medical Center Groningen (UMCG) is a 1300-bed tertiary center and has a 27-bed haematology ward. This ward has four 4 patient rooms, one double room and nine private rooms. Patients were identified by a search of the laboratory electronic database for all blood cultures between September 2005 and September 2010 from the haematology ward. In this period a total of 1086 patients were admitted to the haematology ward of whom 672 blood cultures were taken. (Figure 1) Case patients were identified by a search for all blood cultures positive for E. faecium. Of each patient with an $E$. faecium blood culture, the first positive blood culture was selected: a total of 33 patients with E. faecium blood cultures were identified. For the main purpose of our study, (an algorithm to decide whether or not to add glycopeptides to the initial empirical antibiotic therapy at the moment a blood culture is taken) we choose to use a selection of all the patients of which a blood culture was taken (positive as well as negative), except those with E. faecium blood culture $(n=672-33=639)$. After all, this whole group had the same grounds to obtain a blood culture at the (retrospective) moment the blood culture was drawn. This would also be the case in prospective situations where this algorithm could be applied on. A total of 66 control patients were randomly selected: first a patient was randomly selected; subsequently a blood culture was randomly selected. Patients were not matched for age or sex.

\section{Data collection}

Patient data were gathered by reviewing hospital electronic records and stored hard-copy records. The date the blood culture was taken was chosen as day 0 and from that point all data were reviewed all data retrospectively. Clinical data collected included information of underlying disease, admission status, co-morbidities, neutropenia, C-reactive protein (CRP) levels, fever and signs of organ failure prior to blood culture. Microbiological data collected included clinical source of infection, information about E. faecium colonization and antibiotic use 30 days prior to positive blood culture. If a patient had diarrhea, records were also reviewed for Clostridium difficile. Antibiotic susceptibility patterns, presence of polymicrobial bacteremia and positive galactomannan tests were gathered. Antibiotic treatment with vancomycin or teicoplanin for E. faecium bacteremia was evaluated. Outcomes were measured by need of ICU admission and mortality at 7 and 30 days after blood culture.

\section{Clinical notifications and definitions}

During the retrospective study period, blood cultures were drawn for neutropenic fever or other clinical signs for infection. Fever was defined as temperature $>38.5^{\circ} \mathrm{C}$ or $>$ $38^{\circ} \mathrm{C}$ for 24 hours was a reason for further examination. An absolute neutrophil count below $0.5 \times 10^{9} / \mathrm{L}$ 


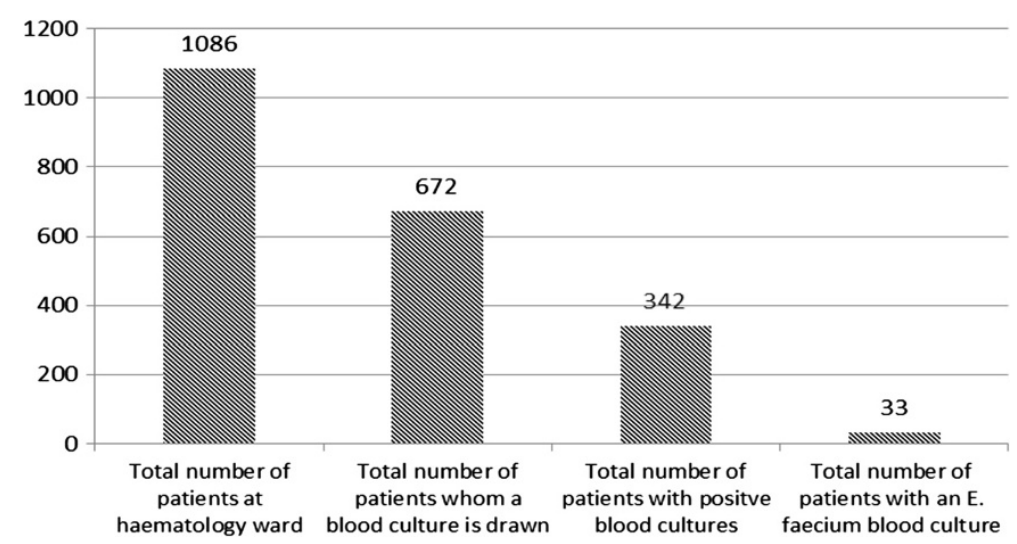

Figure 1 Patients at the haematology ward of the UMCG during the period September 2005-September 2010: Thirty-three of the patients with positive blood cultures (672) had an E. faecium blood culture ( 5\%).

was defined as neutropenia. For organ failure the following definitions were used: renal failure was defined as creatinin $>176 \mu \mathrm{mol} / \mathrm{L}$, hepatic failure as bilirubin $>43 \mathrm{mmol} / \mathrm{L}$ and pulmonary failure as bilateral lung infiltrates or signs of acute respiratory distress syndrome (ARDS). These definitions were according to guidelines used for defining organ failure in severe sepsis [13]. Polymicrobial infection was defined as a micro-organism other than E. faecium within \pm 7 days of the blood culture. For the controls it was defined as an additional microorganism within \pm 7 days of a positive blood culture. In this definition less pathogenic micro-organisms such as CoNS, Corynebacteriae, Micrococcus spp. and Bacillus spp. as an additional micro-organism were excluded.

\section{Infection prevention regimen haematology ward}

At the haematology ward of our hospital, selective decontamination of the digestive tract (SDD) is performed in patients with an (expected) reversible neutropenia or increased risk of infection. The implementation is as follows: surveillance cultures from faeces, throat and urine at admission day, then once a week only faeces and throat cultures during the duration of neutropenia. Penicillin (to prevent streptococcal sepsis) and ciprofloxacin or cotrimoxazol or colistine or tobramycin (orally) are used as prophylactic antibiotics depending on the resistance pattern of surveillance cultures. Amphoterin B, nystatin or fluconazole are given orally as antifungal therapy. The choice of empirical antibiotic therapy is piperacillin-tazobactam.

Screening for E. faecium in this period was done on BME(G) agar plates. This contained Meropenem $64 \mathrm{mg} / \mathrm{L}$, Oxacillin $10 \mathrm{mg} / \mathrm{L}$, Amphotericin-B $20 \mathrm{mg} / \mathrm{L}$ and esculin. Hereby we screened for ampicillin resistant $E$. faecium (HA E. faecium). From January 2007 these agar plates also contained gentamicin $128 \mathrm{mg} / \mathrm{L}$ since there was an increase of high level gentamicin resistant E. faecium in our hospital from that time period.

\section{Identification and susceptibility testing}

Blood cultures were performed using the BACTEC system (Becton Dickinson $\left.{ }^{\text {ts }}\right)$. Further determination and susceptibility testing were performed for gram positive streptococci that were catalase negative and PYR positive. As for E. faecium surveillance cultures, only colonies that grew on the BMEG plates with black borders were further determined. Species were identified using the VITEK $^{\circ} 2$ System (BioMérieux ${ }^{\text {min }}$ ) or API20 Strep System (BioMérieux ${ }^{\mathrm{Tm}}$ ). Subsequently antimicrobial susceptibility testing was performed using the VITEK $^{\circ} 2$ System or disk diffusion tests respectively.

\section{Statistical analyses}

Statistical analyses were performed using SPSS for Windows, rel 18.0. Univariate analyses were performed using the Fisher's exact or Chi-square methods for categorical variables. The Student's t-test or Mann-Whitney $U$-test was used for the continuous variables. Results with a $p$-value of $\leq 0.05$ were considered to be statistically significant. All $p$-values are two-tailed. Significant variables were used in the multivariate logistic regression.

\section{Deriving prediction model from a nested case-control design}

To overcome the overestimation of risks because of overrepresentation of cases, we choose to perform a nested case-control design where the cases represent $5 \%$ and controls $95 \%$ of the whole population (Figure 1). Therefore the following factor to the intercept of the logistic regression model is added: $\mathrm{c}=\ln \left(\mathrm{q}_{0} /\left(1-\mathrm{q}_{0}\right)\right)$, whereas $\mathrm{q}_{0}$ is the true prevalence of the diseases in the population. With this correction the risk of an individual to get the disease can be estimated by the formula 
$\mathrm{e}^{\beta 0+c+\beta 1 X 1+\ldots \beta \mathrm{kXk}} / 1+\mathrm{e}^{\beta 0+\mathrm{c}+\beta 1 \mathrm{X} 1+\ldots \beta \mathrm{kXk}}$. In this formula, $\beta_{0}$ is the intercept from the linear regression equation, $\beta_{1} /$ $\beta_{\mathrm{k}}$ is the regression coefficient derived from the multivariate logistic regression and $\mathrm{X}_{1} / \mathrm{X}_{\mathrm{k}}$ is the value of the predictor. In this study, $\mathrm{q} 0$ is the prevalence of patients with an E. faecium blood culture. Since we were only interested in those patients of whom a blood culture is drawn, $\mathrm{c}=\ln$ $(0.05 /(1-0.05))=-2.94$. Controls should be a random selection representative of the population [14] which is the case since we randomly selected the 66 control patients.

\section{Results \\ Patients}

A total of 99 patients were evaluated: 33 cases (E. faecium) and 66 controls. Characteristics of the 66 controls showed the following blood culture results: $E$. coli $(\mathrm{n}=4)$, Streptococcus viridans $(\mathrm{n}=2)$, CoNS $(\mathrm{n}=4)$, Corynebacterium spp. $(\mathrm{n}=1)$ and no growths $(\mathrm{n}=55)$. Comparisons of the demographic and clinical data are presented in Table 1 . There were no significant differences between type or status of disease. Patients with E. faecium bacteremia were associated with higher age and longer hospitalization days prior to blood culture as well as one year before admission. They were also associated with severe and longer duration of neutropenia, longer duration of fever and higher CRP levels at time of blood culture withdrawal. Penicillin and quinolones as a part of the SDD regimen and piperacillin-tazobactam as empirical broad-spectrum antibiotics were the most frequently used antibiotics; however this did not differ between the two groups. Only "other" antibiotics were more frequently given in the E. faecium group. This was mainly colistine, a polymixin antibiotic, though colistine use alone was not significant.

\section{Microbes}

From the 33 cases, fourteen patients (42.4\%) had a single blood culture, nineteen (57.6\%) had more than one blood culture and 11 (33.3\%) had more than two blood cultures. All E. faecium blood isolates were resistant to amoxicillin. No VRE strains were identified in this study. High-level gentamicin resistance (HLGR) was found in 19 (57.6\%) of the 33 E. faecium blood isolates. Three of the 19 patients with HLGR E. faecium also had low level gentamicin resistant E. faecium in their blood cultures (multiple blood cultures).

Comparisons of the microbial data are presented in Table 2. Polymicrobial infections were found in $9.1 \%$ of the cases compared to $1(1.5 \%)$ in the control group ( $p=0.107)$. Pathogens isolated were Clostridium perfringens, Pseudomonas aeruginosa, and Streptococcus species. Three case patients $(9.1 \%)$ had a positive Galactomannan compared to $2(3.0 \%)$ in the control group $(p=0.330)$.
An abdominal focus was found to be associated with $E$. faecium bacteremia $(p=0.003)$ of which diarrhea appeared to be most distinct variable. Only one patient with an $E$. faecium BSI had a positive Clostridium difficile toxin test (no $C$. difficile in stool culture) at time of diarrhea. This was two days prior to the positive blood culture, together with a positive $E$. faecium faeces culture, though this patient was already colonized with E. faecium for several weeks.

Patients with E. faecium BSI were more often detected to be colonized with E. faecium prior to blood culture $(p=<0.001)$. A total of twenty-one patients $(63.6 \%)$ were colonized with E. faecium prior to the positive blood culture with a median of 1 (range 0-8), compared to $24.2 \%$ in the control group with a median of 0 (range 0-6). Twelve patients (36.4\%) were not found to be colonized with the surveillance cultures. However, nine of these twelve patients had a blood culture with low level gentamicin resistant E. faecium. Seven of these twelve patients $(58.3 \%)$, had a positive faeces culture with $E$. faecium after all within 30 days after positive blood culture; five with high level gentamicin resistant $E$. faecium, two with low level gentamicin resistant $E$. faecium. The majority of the patients (69.7\%) were still colonized up to 30 days after the first positive blood culture. This includes both patients that were already colonized and patients who had a positive culture with E. faecium within 30 days after their positive blood culture.

\section{Outcomes and treatment}

Both groups had an equal antibiotic treatment for piperacillin-tazobactam as well as for glycopeptide treatment at time of blood culture withdrawal. (Table 3) Patients with an E. faecium BSI were more often admitted to the ICU after the positive blood culture. Reasons for ICU admissions were predominantly sepsis, mostly with an abdominal focus (abdominal sepsis). The 7-day mortality as well as the 30-day mortality were significantly higher in patients with E. faecium BSI compared to the control group $(30.3 \%$ vs $4.5 \% ; p=0.001$ and $39.4 \%$ vs $10.6 \% ; p=0.001$ respectively). All 10 patients with $E$. faecium BSI that died within 7 days after their last positive culture were diagnosed with sepsis or severe infection, six of them $(60 \%)$ had an clear abdominal focus (abdominal sepsis). Another three patients died after 30 days, one diagnosed with a septic shock, the other two patients had multiple diagnoses.

More detailed data considering antibiotic treatment in patients with an E. faecium BSI including mortality rates are presented in Table 4 . Only 4 patients (12.1\%) received glycopeptide treatment at time of blood culture withdrawal. Three of them had an empirical treatment and one received treatment because of an earlier proven 
Table 1 Comparison of demographic and clinical characteristics of cases (E. faecium) and controls

Demographics

Age, mean $\pm S D$, years

Type of malignancy: ${ }^{\text {a }}$

Leukaemia (AML, MDS, ALL) for chemotherapy

Leukemia for allogeneic stem cell transplantation

Lymphoma's, Kahler, CLL and others undergoing autologous stem cell transplantation

Lympfhoma's, Kahler, CLL not undergoing autologous stem cell transplantation

\section{Status of disease:}

Remission

Not in remission ${ }^{b}$

Relapse

Reason for admission:

Infection

Chemotherapy

Stem cell transplantation ${ }^{c}$

Length of hospital stay:

Length in days prior to positive blood culture, median (range)

Length in days 1 year before admission, median (range)

Signs of organ failure: ${ }^{d}$

Renal (creatinine $>176 \mu \mathrm{mol} / \mathrm{L})$

Hepatic (bilirubin $>34 \mathrm{mmol} / \mathrm{L}$ )

Lung (bilateral lung infiltrates)

Days of fever, median (range) ${ }^{d}$

\section{Neutropenia:}

Neutropenia $<0.1 \times 10^{9} / \mathrm{L}^{\mathrm{e}}$

Neutropenia $<0.5 \times 10^{9} / \mathrm{L}^{\mathrm{e}}$

Neutropenia $<2.0 \times 10^{9} / \mathrm{L}^{\mathrm{e}}$

Duration of neutropenia $<0.5 \times 10^{9} / \mathrm{L}$ prior to blood culture, median (range)

\section{CRP (C-reactive protein in $\mathrm{mg} / \mathrm{L}$ ):}

Levels 7 days prior to blood culture, median (range)

Levels at time of blood culture, median (range)

At time of blood culture CRP $>125 \mathrm{mg} / \mathrm{L}$

Antibiotic therapy at time of blood culture and/or 30 days before:

Penicillins

Cotrimoxazole

Quinolones

Cephalosporins

Carbapenems

Others $^{f}$

Cases $(\boldsymbol{n}=\mathbf{3 3})$

$18(68.2 \%)$

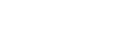

19 (57.6\%)

$2(6.1 \%)$

$6(18.2 \%)$

$6(18.2 \%)$

9 (27.3\%)

$24(72.7 \%)$

$7(21.2 \%)$

$4(12.1 \%)$

$21(63.6 \%)$

$8(24.2 \%)$

$21(2-52)$

$43(6-131)$

$2(6.1 \%)$

2 (6.1\%)

$4(12.1 \%)$

$2(0-7)$

\section{$20(60.6 \%)$}

$28(84.8 \%)$

$29(87.9 \%)$

$8.0(0-27)$

26 (3-263)

188 (7-288)

23 (69.7\%)

24 (72.7\%)

$12(36.4 \%)$

$25(75.8 \%)$

$6(18.2 \%)$

$6(18.2 \%)$

$19(57.6 \%)$
Controls $(n=66)$

p-value

45 (54.5\%)

$52.2 \pm 9.1$

0.378

$28(42.6 \%)$

2 (3.0\%)

17 (25.8\%)

19 (28.8\%)

1 (16.7\%)

0.215

55 (83.3\%)

0.215

14 (21.2\%)

1.000

0.476

$13(19.7 \%)$

34 (51.5\%)

19 (28.8\%)

$13.5(1-84)$

0.007

$24(1-133)$

0.018

0.746

0.109

$0(0.0 \%)$

0.769

10 (15.2\%)

0.001

0.002

19 (28.8\%)

$<0.001$

28 (42.4\%)

0.004

$39(59.1 \%)$

$<0.001$

$0.0(0-26)$

0.07

47 (5-347)

0.006

108 (3-426)

0.002

24 (36.4\%)

$40(60.6 \%) \quad 0.234$

$18(27.3 \%) \quad 0.353$

$51(77.3 \%) \quad 0.866$

$4(6.1 \%) \quad 0.079$

$5(7.6 \%) \quad 0.113$

$16(24.2 \%)$

0.001

${ }^{\mathrm{a}} A M L$ acute myeloid leukaemia, MDS myelodysplastic syndrome, ALL acute lymphoblastic leukaemia, CLL chronic lymphoblastic leukaemia. ${ }^{\mathrm{b}}$ Including patients partially in remission. 'Allogeneic as well as autologous stem cell transplantation. ${ }^{\mathrm{d}}$ At the day of blood culture till 7 days prior to blood culture. ${ }^{\mathrm{e}}$ At the day of blood culture withdrawal ${ }^{f}$ colistin, tetracyclin, macrolides, aminoglycosides, metronidazole.

CoNS infection. After 24 hours a total of 19 patients (57.6\%) received glycopeptide treatment. Of these 19 cases, four were empirically treated upfront because of septic profile, two cases because of a CoNS infection and 13 cases recommended by the medical microbiologist because of suspected or proven E. faecium blood 
Table 2 Comparison of the microbiological characteristics of cases (E. faecium) and controls

\begin{tabular}{|c|c|c|c|}
\hline & Cases $(n=33)$ & Controls $(n=66)$ & $p$-value \\
\hline \multicolumn{4}{|l|}{ 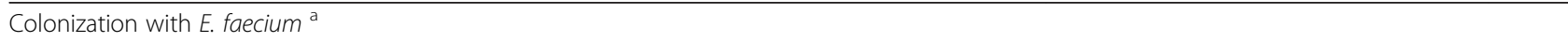 } \\
\hline 7 days prior to blood culture & $13(39.4 \%)$ & $10(15.2 \%)$ & 0.007 \\
\hline 30 days prior to blood culture & $19(57.6 \%)$ & $14(21.2 \%)$ & $<0.001$ \\
\hline 90 days prior to blood culture & $21(63.6 \%)$ & $16(24.2 \%)$ & $<0.001$ \\
\hline Number of faeces cultures with E. faecium 30 days prior to blood culture, median (range) & $1(0-8)$ & $0(0-6)$ & $<0.001$ \\
\hline \multicolumn{4}{|l|}{ Type of blood culture } \\
\hline Polymicrobial b & $3(9.1 \%)$ & $1(1.5 \%)$ & 0.107 \\
\hline Galactomannan & $3(9.1 \%)$ & $2(3.0 \%)$ & 0.330 \\
\hline \multicolumn{4}{|l|}{ Clinical source of infection } \\
\hline Abdominal focus: abdominal pain and/or diarrhea & $25(75.8 \%)$ & $29(43.9 \%)$ & 0.003 \\
\hline Abdominal pain & $9(27.3 \%)$ & $11(16.7 \%)$ & 0.215 \\
\hline Diarrhea & $23(69.7 \%)$ & $26(39.4 \%)$ & 0.004 \\
\hline Mucositis & $13(39.4 \%)$ & $18(27.3 \%)$ & 0.220 \\
\hline \multicolumn{4}{|l|}{ Lungs } \\
\hline Coughing and/or sputum & $8(24.2 \%)$ & $15(22.7 \%)$ & 0.866 \\
\hline Radiological proof of pneumonia or lung infiltrates & $4(12.1 \%)$ & $14(21.2 \%)$ & 0.269 \\
\hline Ear Nose Throat & $1(3.0 \%)$ & $2(3.0 \%)$ & 1.000 \\
\hline Skin & 7 (21.2\%) & $19(28.8 \%)$ & 0.419 \\
\hline Urinary infection & $1(3.0 \%)$ & $9(13.6 \%)$ & 0.158 \\
\hline
\end{tabular}

an faeces culture, part of the SDD regimen. ${ }^{b}$ Within \pm 7 days, less pathogenic micro-organisms (coagulase-negative staphylococci, corynebacteria, micrococcus spp. and bacillus spp.) are excluded.

culture. Still, fourteen patients (42.4\%) had no adequate treatment for their infection after 24 hours.

Additional statistical analyses were performed on patients with an E. faecium BSI (cases) to determine additional risk factors for mortality. Only the numbers of blood cultures were found to be statistically significant for mortality at 7 days, with significant trend effect in case of more positive blood cultures. (Additional file 1) None of the other demographic, clinical or microbiologic factors listed in Tables 1 and 2 (e.g. neutropenia, mucositis, glycopeptide treatment) were found to be additional risk factors.

Multivariable regression analysis and prediction modeling Variables included in the multivariate regression analyses are shown in Table 5. Independent risk factors for an
E. faecium BSI are colonization with E. faecium 30 days prior to blood culture (OR 5.71; CI 1.7-18.7), combination of neutropenia and abdominal focus (4.37; 1.4-13.4), age $>58$ years $(4.01 ; 1.3-12.5)$, hospital stay prior to blood culture $>14$ days $(3.55 ; 0.98-12.9)$ and CRP (C-reactive protein) level $>125 \mathrm{mg} / \mathrm{L}$ (4.37; 1.1-10.2).

Subsequently these independent risk factors were used in order to develop the prediction model. A subset of this prediction model is shown in Table 6. Hereby the formula $e^{\beta 0+c+\beta 1 X 1+\ldots \beta k X k} / 1+e^{\beta 0+c+\beta 1 X 1+\ldots \beta k X k}$ was used, whereas $\beta$ was deduced from the multivariate regression analysis as shown in Table 5 . Since five variables were tested and used in this model, a total of 32 outcomes are possible. If a patient has all the five variables at the moment of blood culture withdrawal, the risk of an E. faecium BSI is $47.5 \%$. If a patient has none of the

Table 3 Comparison of outcome and antibiotic treatment of cases (E. faecium) and controls

\begin{tabular}{|c|c|c|c|}
\hline & Cases $(n=33)$ & Controls $(n=66)$ & $p$-value \\
\hline Piperacillin-tazobactam treatment at time blood culture is drawn and/or 30 days before & $22(66.7 \%)$ & $42(63.6 \%)$ & 0.766 \\
\hline Vancomycin/teicoplanin treatment at time of blood culture withdrawal & $4(12.1 \%)$ & $8(12.1 \%)$ & 1.000 \\
\hline ICU admission till 7 days after positive bloodculture & $5(15.2 \%)$ & $1(1.5 \%)$ & 0.015 \\
\hline \multicolumn{4}{|l|}{ Mortality $^{*}$} \\
\hline At 7 days & $10(30.3 \%)$ & $3(4.5 \%)$ & 0.001 \\
\hline At 30 days & $13(39.4 \%)$ & $7(10.6 \%)$ & 0.001 \\
\hline
\end{tabular}

"After last positive blood culture with E. faecium. 
Table 4 Antibiotic treatment with vancomycin or teicoplanin in patients with E. faecium BSI, including mortality rates $(n=33)$

\begin{tabular}{|c|c|c|c|c|c|c|}
\hline & \multicolumn{6}{|c|}{ Vancomycine/teicoplanin treatment cases $(n=33)$} \\
\hline & \multicolumn{4}{|c|}{ Yes } & \multicolumn{2}{|c|}{ No } \\
\hline & Empirical & Mortality & Therapeutic & Mortality & & Mortality \\
\hline \multirow{2}{*}{ At time of blood culture withdrawal } & 3 & $2 / 3$ & $1^{*}$ & 0 & 29 & $11 / 29$ \\
\hline & $(9.1 \%)$ & $(66.7 \%)$ & $(3 \%)$ & $(0 \%)$ & (87.9\%) & $(37.9 \%)$ \\
\hline \multirow{2}{*}{ After $24 \mathrm{hrs}$} & 4 & $3 / 4$ & $13+2^{*}$ & $5 / 15$ & 14 & $5 / 14$ \\
\hline & $(12.1 \%)$ & $(75 \%)$ & $(45.5 \%)$ & (33.3\%) & $(42.4 \%)$ & $(35.7 \%)$ \\
\hline
\end{tabular}

*Because of coagulase negative staphylococci.

variables the risk is close to zero. In clinical decision making the clinician can fill in the variables; 0 for a negative and 1 for a positive score and thereby deduce the risk of E. faecium BSI. (All 32 variables and probabilities are available in an Additional file 1).

\section{Discussion}

Nowadays E. faecium has become an emerging and challenging pathogen in hospitals and even more has replaced $E$. faecalis as the predominant enterococcus species [8]. The increase of E. faecium BSIs in our study are in line with the numbers of a recent EARSS (European Antimicrobial Resistance Surveillance System) study, in which E. faecium increased most significant in BSIs compared to other major pathogens [15].

All E. faecium strains from the blood cultures in our study belonged to the HA/CC-17 strains. They were all amoxicillin (ampicillin) resistant and insertion sequence 16 (IS16) positive, which is a marker for these strains [16]. HA/CC-17 strains seem to be successful in acquiring accessory virulence and antibiotic genes and therefore might set the stage for VRE [17]. In vancomycin resistant $E$. faecium infections, adequate treatment of serious infections becomes limited. Although some novel antimicrobials such as linezolid and daptomycin have been developed, these also have their limitations; moreover resistance to these antimicrobials has already been described [18].

In line with previous studies prior colonization with HA E. faecium showed to be an independent risk factor for E. faecium BSI $[19,20]$. This study showed that the majority of patients $(63.6 \%)$ were first colonized prior to the development of E. faecium BSI; moreover it seemed to be the most important/significant independent risk factor for E. faecium BSI in our study. It is important to keep in mind that multiple swabs might be needed to detect the majority of carriers [21] and E. faecium can persist for a long period [22] which is also seen in our study. Environmental contamination and person-toperson spread are factors contributing to the acquisition of E. faecium [23,24]. Enterococcus spp. are quite tenacious and may survive for more than 4 months under dry conditions [25]. Therefore standard hygiene (e.g. hand hygiene) and appropriate infection-control measures (e.g. risk surface disinfection) are essential.

Neutropenia and abdominal focus (diarrhea and/or abdominal pain) were also associated with $E$. faecium BSI. Because these variables seem to be related to each other, as they individually excluded each other in regression analysis, the two variables were combined. The extensive chemo- and transplantation therapy the patients receive is often associated with neutropenia and diarrhea [26]. In case of severe neutropenia or chemotherapy induced diarrhea which can be seen as injury of the mucosal barrier, $E$. faecium has the opportunity to enter the bloodstream.

Subsequently we expected mucositis, which relates more to the oral toxicity of chemotherapy, to be an associated variable. Kuehnert et al. showed that the risk of VRE BSI increased with increasingly severe mucositis [27]. In contrast, Worth et al. didn't find mucositis to be associated with $E$. faecium infection; however it hadn't a well-validated mucositis severity index [28]. Perhaps a more validated mucositis stratification would have shown other results in our study.

Table 5 Multivariate logistic regression analyses: risk factors associated with an $E$. faecium BSI $(n=33)$

\begin{tabular}{|c|c|c|c|c|}
\hline Variables tested & B & $p$ & OR & {$[95 \% \mathrm{Cl}]$} \\
\hline A. Colonization with E. faecium 30 days prior to blood culture & 1.742 & 0.004 & 5.71 & {$[1.7-18.7]$} \\
\hline B. Neutropenia and abdominal focus ${ }^{*}$ & 1.474 & 0.010 & 4.37 & {$[1.4-13.4]$} \\
\hline C. Age $>58$ years & 1.390 & 0.017 & 4.01 & {$[1.3-12.5]$} \\
\hline D. Days of admission prior to blood culture $>14$ days & 1.267 & 0.054 & 3.56 & {$[0.98-12.9]$} \\
\hline E. CRP $>125 \mathrm{mg} / \mathrm{L}$ & 1.216 & 0.032 & 4.37 & {$[1.1-10.2]$} \\
\hline
\end{tabular}

${ }^{*}$ Abdominal pain and/or diarrhea. $\mathrm{B}=$ regression coefficient. $P p$-value. OR Odds ratio. 95\% Cl 95\% confidence interval. 
Table 6 Prediction model to determine the risk of $E$. faecium BSI (subset)

\begin{tabular}{cccccc}
\hline A & B & C & D & E & Probability \\
\hline 1 & 1 & 1 & 1 & 1 & 47.5 \\
1 & 1 & 1 & 1 & 0 & 21.2 \\
1 & 1 & 1 & 0 & 1 & 20.3 \\
1 & 1 & 0 & 1 & 1 & 18.4 \\
1 & 0 & 1 & 1 & 1 & 17.2 \\
0 & 1 & 1 & 1 & 1 & 13.7 \\
0 & 0 & 0 & 0 & 0 & 0.08 \\
\hline \multicolumn{7}{l}{ For this prediction model the formula $\mathrm{e}^{\beta 0+c+\beta 1 \times 1+\ldots \beta k \mathrm{k} / 1+\mathrm{e}^{\beta 0+c+\beta 1 \times 1+\ldots \beta k \times k}}$}
\end{tabular}

For this prediction model the formula $\mathrm{e}^{\beta 0+c+\beta 1 \times 1+\ldots \beta \mathrm{k} \times \mathrm{k}} / 1+\mathrm{e}^{\beta 0+c+\beta 1 \times 1+\ldots \beta \mathrm{k} \times \mathrm{k}}$
was used, whereas $\beta$ was deduced from the multivariate regression analysis as shown in Table 5.0 variable absent, 1 variable present $A$ Colonization with $E$. faecium 30 days prior to blood culture $B$ Neutropenia and abdominal focus (diarrhea or abdominal pain) $C$ Age over 58 years $D$ Days of admission prior to blood culture more than 14 days $E$ CRP $>125 \mathrm{mg} / \mathrm{L}$.

CRP level and fever as infection parameters were both found to be significant. However, they individually excluded each other in the regression analysis. Therefore we chose to include CRP level in our model as it is a more objective parameter. Especially in these haematology patients, fever can be aspecifically related to for example drug fever or inflammation like mucositis.

Not many studies have identified age to be an independent risk factor. However the majority of the patients with E. faecium infections in the studies are at higher age (50-70 years) and these studies included a more specific control group $[11,29,30]$ whereas we choose a random selection representative for the total population of the haematology ward during the study period.

Since E. faecium is considered to be a nosocomial pathogen, a prolonged hospital length of stay as a predictor in E. faecium bacteremia is as we expected. For VRE as a multi-resistant pathogen it is clear it is associated with a longer hospital length of stay. Though also for vancomycin-susceptible (VSE), but ampicillin resistant E. faecium (ARE) as in our study, this association had been shown [31,32].

Another risk factor often associated with E. faecium infection is previous antibiotic use [30]. Moreover, numbers of enterococci in SDD increases since they are not covered [33]. We haven't found a strong association between antibiotic use and an E. faecium BSI, since the majority of both patient groups received SDD.

Additional analysis between patients with and without an E. faecium BSI did not result in additional risk factors for mortality besides the total number of positive $E$. faecium blood cultures. However numbers were often too small to perform adequate statistical analyses between the two groups.

This study has some limitations. Firstly, the data was retrospectively gathered. Although both stored-hard copy and electronic records were reviewed, for certain clinical parameters precise monitoring was difficult. Secondly, this is a single-centre study whereas local epidemiological variables and infection prevention measures must be considered. Thirtly, from January 2007 surveillance cultures were screened for meropenem and high level gentamicin resistant E. faecium. The reason for this was an increase in E. faecium of which the major part was high level gentamicin resistant in our hospital from that time period. An unknown number of $E$. faecium of gentamicin susceptible surveillance cultures have been missed during this period. However, we still detected some gentamicin low level resistant E. faecium $(5 / 200$ patients $\sim 5 \%)$ from that time period. From February 2011 we use $2 \mathrm{mg} / \mathrm{L}$ gentamicin in our BMEG screening agars instead of $128 \mathrm{mg} / \mathrm{L}$. Hereby we see an increase of $\sim 30 \%$ due to low level gentamicin E. faecium in the haematology ward for the period February 2011 July 2013. However, there seems to be a shift again from 2012, whereas gentamicin high level E. faecium, accounts for up to $80 \%$ of the HA E. faecium both in screening cultures as well as in blood cultures for the period February 2012 - July 2013. This should be taken into account considering results of E. faecium colonization in our study. It is difficult to assess the implication of this limitation on the prediction model with respect to the odds ratio. Moreover, patients can have several $E$. faecium strains in their surveillance cultures as well as in blood cultures. Finally the majority of our control group had blood cultures with 'no growths'. This might have several reasons, for example patients could have had fever due to the malignancy or drug fever or inflammation because of mucositis. It could also partially be explained by the fact patients received SDD. One can state that these patients had a lower degree of illness, compared to patients with an E. faecium blood culture. However, retrospective circumstances for both groups were equal. Both groups had the same grounds to obtain a blood culture; neutropenic fever or other clinical signs of infection. Also for the purpose of the study, a prediction model in order to decide whether or not add glycopeptide to the empirical antibiotic treatment at the moment a blood culture is drawn, we choose to select this group of patients as controls.

In conclusion this study demonstrated that colonization with HA E. faecium 30 days prior to blood culture, combination of neutropenia and abdominal focus, age > 58 years, hospital stay prior to blood culture $>14$ days and CRP level $>125 \mathrm{mg} / \mathrm{L}$ are independent risk factors for E. faecium BSI. In agreement with previous studies, this study showed that E. faecium infections can cause severe infections and are associated with high mortality rates in patients with haematologic malignancies [10,34]. Thereby risk stratification becomes necessary in those haematology patients at high risk. Using a prediction 
model for risk stratification as designed in this study, antibiotic stewardship in terms of prudent use of glycopeptides becomes possible. Together with infection control measures this might be helpful controlling further increase of VRE. The prediction model in this study is based on one specific haematology ward, though it would be worthwhile to verify this prediction model in a prospective multicenter study.

\section{Additional file}

\section{Additional file 1: Table S1. Association between numbers of $E$.} faecium blood cultures and mortality in patients with an E. faecium BSI/ cases $(n=33)$. Table S2. Complete prediction model to determine the risk of E. faecium BSI.

\section{Competing interest}

The authors declare that they have no competing interests.

\section{Authors' contributions}

XZ has contributed to the conception and design of the study, gathered laboratory and clinical data, analyzed the data and drafted the original article. JA contributed to the conception and design of the study, gathered laboratory data and revised the article. LS contributed to the conception and design of the study and critically revised the article. AF critically revised the article. All authors read and approved the final manuscript.

\section{Acknowledgements}

This work was supported by the Interreg IVa-funded projects EurSafety Heath-net (III-1-02 = 73) and SafeGuard (III-2-03=025), part of a DutchGerman cross-border network supported by the European Commission, the German Federal States of Nordrhein-Westfalen and Niedersachsen, and the Dutch provinces of Overijssel, Gelderland, and Limburg.

We thank I.M. Nolte (University Medical Center Groningen, Department of Epidemiology) and T. Donker (University Medical Center Groningen, Department of Medical Microbiology) for the excellent help and discussion about the statistical analysis of the data. We thank G.A. Kampinga for the great help and knowledge on microbiological methods used at the laboratory.

\section{Author details}

'Department of Medical Microbiology, University of Groningen, University Medical Center Groningen, Groningen, The Netherlands. ${ }^{2}$ Department of Haematology, University of Groningen, University Medical Center Groningen, Groningen, The Netherlands.

Received: 28 May 2013 Accepted: 1 September 2013

Published: 11 September 2013

\section{References}

1. Arias CA, Murray BE: The rise of the Enterococcus: beyond vancomycin resistance. Nat Rev Microbiol 2012, 10(4):266-278.

2. Leclercq R, Canton R, Brown DF, Giske CG, Heisig P, Macgowan AP, Mouton JW, Nordmann P, Rodloff AC, Rossolini GM, Soussy CJ, Steinbakk M, Winstanley TG, Kahlmeter G: EUCAST expert rules in antimicrobial susceptibility testing. Clin Microbiol Infect 2013, 19:141-160.

3. Jett BD, Huycke MM, Gilmore MS: Virulence of enterococci. Clin Microbiol Rev 1994, 7(4):462-478.

4. Tornieporth NG, Roberts RB, John J, Hafner A, Riley LW: Risk factors associated with vancomycin-resistant Enterococcus faecium infection or colonization in 145 matched case patients and control patients. Clin Infect Dis 1996, 23(4):767-772.

5. Rice LB: Emergence of vancomycin-resistant enterococci. Emerg Infect Dis 2001, 7(2):183-187.

6. Werner G, Coque TM, Hammerum AM, Hope R, Hryniewicz W, Johnson A, Klare I, Kristinsson KG, Leclercq R, Lester CH, Lillie M, Novais C, Olsson-Liljequist B, Peixe LV, Sadowy E, Simonsen GS, Top J, VuopioVarkila J, Willems RJ, Witte W, Woodford N: Emergence and spread of vancomycin resistance among enterococci in Europe. Euro Surveill 2008, 13(47):19046.

7. Leavis HL, Bonten MJ, Willems RJ: Identification of high-risk enterococcal clonal complexes: global dispersion and antibiotic resistance. Curr Opin Microbiol 2006, 9(5):454-460.

8. Top J, Willems R, Blok H, de Regt M, Jalink K, Troelstra A, Goorhuis B, Bonten M: Ecological replacement of Enterococcus faecalis by multiresistant clonal complex 17 Enterococcus faecium. Clin Microbiol Infect 2007, 13(3):316-319.

9. Hoge CW, Adams J, Buchanan B, Sears SD: Enterococcal bacteremia: to treat or not to treat, a reappraisal. Rev Infect Dis 1991, 13(4):600-605.

10. Vydra J, Shanley RM, George I, Ustun C, Smith AR, Weisdorf DJ, Young JA: Enterococcal Bacteremia is associated with Increased Risk of Mortality in Recipients of Allogeneic Hematopoietic Stem Cell Transplantation. Clin Infect Dis 2012, 55(6):764-770.

11. McBride SJ, Upton A, Roberts SA: Clinical characteristics and outcomes of patients with vancomycin-susceptible Enterococcus faecalis and Enterococcus faecium bacteraemia--a five-year retrospective review. Eur J Clin Microbiol Infect Dis 2010, 29(1):107-114.

12. Suppli M, Aabenhus R, Harboe ZB, Andersen LP, Tvede M, Jensen JU: Mortality in enterococcal bloodstream infections increases with inappropriate antimicrobial therapy. Clin Microbiol Infect 2011 17:1078-1083.

13. Dellinger RP, Levy MM, Carlet JM, Bion J, Parker MM, Jaeschke R, Reinhart K, Angus DC, Brun-Buisson C, Beale R, Calandra T, Dhainaut JF, Gerlach $\mathrm{H}$, Harvey M, Marini JJ, Marshall J, Ranieri M, Ramsay G, Sevransky J, Thompson BT, Townsend S, Vender JS, Zimmerman JL, Vincent JL, International Surviving Sepsis Campaign Guidelines Committee, American Association of Critical-Care Nurses, American College of Chest Physicians, American College of Emergency Physicians, Canadian Critical Care Society, European Society of Clinical Microbiology and Infectious Diseases, European Society of Intensive Care Medicine, et al: Surviving Sepsis campaign: International guidelines for management of severe sepsis and septic shock: 2008. Crit Care Med 2008, 36(1):296-327.

14. Anderson JA: Separate sample logistic discrimination. Biometrika 1972 59:19-35.

15. de Kraker ME, Jarlier V, Monen JC, Heuer OE, van de Sande N, Grundmann $H$ : The changing epidemiology of bacteraemias in Europe: trends from the European Antimicrobial Resistance Surveillance System. Clin Microbiol Infect 2013, 19:860-868.

16. Werner G, Fleige C, Geringer U, van Schaik W, Klare I, Witte W: IS element IS16 as a molecular screening tool to identify hospital-associated strains of Enterococcus faecium. BMC Infect Dis 2011, 11:80

17. Top J, Willems R, Bonten M: Emergence of CC17 Enterococcus faecium: from commensal to hospital-adapted pathogen. FEMS Immunol Med Microbiol 2008, 52(3):297-308.

18. Theilacker C, Jonas D, Huebner J, Bertz H, Kern WV: Outcomes of invasive infection due to vancomycin-resistant Enterococcus faecium during a recent outbreak. Infect 2009, 37(6):540-543.

19. Edmond MB, Ober JF, Weinbaum DL, Pfaller MA, Hwang T, Sanford MD, Wenzel RP: Vancomycin-resistant Enterococcus faecium bacteremia: risk factors for infection. Clin Infect Dis 1995, 20(5):1126-1133.

20. Montecalvo MA, Horowitz H, Gedris C, Carbonaro C, Tenover FC, Issah A, Cook P, Wormser GP: Outbreak of vancomycin-, ampicillinand aminoglycoside-resistant Enterococcus faecium bacteremia in an adult oncology unit. Antimicrob Agents Chemother 1994, 38(6):1363-1367.

21. Pearman JW: 2004 Lowbury lecture: the Western Australian experience with vancomycin-resistant enterococci - from disaster to ongoing control. J Hosp Infect 2006, 63(1):14-26.

22. Montecalvo MA, de Lencastre $H$, Carraher M, Gedris C, Chung M, VanHorn $K$ Wormser GP: Natural history of colonization with vancomycin-resistant Enterococcus faecium. Infect Control Hosp Epidemiol 1995, 16(12):680-685.

23. de Regt MJ, van der Wagen LE, Top J, Blok HE, Hopmans TE, Dekker AW, Hene RJ, Siersema PD, Willems RJ, Bonten MJ: High acquisition and environmental contamination rates of $\mathrm{CC} 17$ ampicillin-resistant Enterococcus faecium in a Dutch hospital. J Antimicrob Chemother 2008, 62(6):1401-1406.

24. Mascini EM, Troelstra A, Beitsma M, Blok HE, Jalink KP, Hopmans TE, Fluit AC, Hene RJ, Willems RJ, Verhoef J, Bonten MJ: Genotyping and preemptive 
isolation to control an outbreak of vancomycin-resistant Enterococcus faecium. Clin Infect Dis 2006, 42(6):739-746.

25. Wendt C, Wiesenthal B, Dietz E, Ruden H: Survival of vancomycin-resistant and vancomycin-susceptible enterococci on dry surfaces. J Clin Microbiol 1998, 36(12):3734-3736.

26. Benson AB 3rd, Ajani JA, Catalano RB, Engelking C, Kornblau SM, Martenson JA Jr, McCallum R, Mitchell EP, O'Dorisio TM, Vokes EE, Wadler S: Recommended guidelines for the treatment of cancer treatmentinduced diarrhea. J Clin Oncol 2004, 22(14):2918-2926.

27. Kuehnert MJ, Jernigan JA, Pullen AL, Rimland D, Jarvis WR: Association between mucositis severity and vancomycin-resistant enterococcal bloodstream infection in hospitalized cancer patients. Infect Control Hosp Epidemiol 1999, 20(10):660-663.

28. Worth $L$, Thursky KA, Seymour JF, Slavin MA: Vancomycin-resistant Enterococcus faecium infection in patients with hematologic malignancy: patients with acute myeloid leukemia are at high-risk. Eur J Haematol 2007, 79(3):226-233.

29. Conde-Estevez D, Grau S, Albanell J, Terradas R, Salvado M, Knobel H: Clinical characteristics and outcomes of patients with vancomycinsusceptible Enterococcus faecalis and Enterococcus faecium bacteraemia in cancer patients. Eur J Clin Microbiol Infect Dis 2011, 30(1):103-108.

30. Noskin GA, Peterson LR, Warren JR: Enterococcus faecium and Enterococcus faecalis bacteremia: acquisition and outcome. Clin Infect Dis 1995, 20(2):296-301.

31. Coque TM, Willems RJ, Fortun J, Top J, Diz S, Loza E, Canton R, Baquero F: Population structure of Enterococcus faecium causing bacteremia in a Spanish university hospital: setting the scene for a future increase in vancomycin resistance? Antimicrob Agents Chemother 2005, 49(7):2693-2700,

32. Harthug $\mathrm{S}$, Eide GE, Langeland N: Nosocomial outbreak of ampicillin resistant Enterococcus faecium: risk factors for infection and fatal outcome. J Hosp Infect 2000, 45(2):135-144.

33. Benus RF, Harmsen HJ, Welling GW, Spanjersberg R, Zijlstra JG, Degener JE, van der Werf TS: Impact of digestive and oropharyngeal decontamination on the intestinal microbiota in ICU patients. Intensive Care Med 2010, 36(8):1394-1402

34. Todeschini G, Tecchio C, Borghero C, D'Emilio A, Pegoraro E, de Lalla F, Benedetti P, Spolaore P, Pellizzer G: Association between Enterococcus bacteraemia and death in neutropenic patients with haematological malignancies. J Infect 2006, 53(4):266-273.

doi:10.1186/2047-2994-2-24

Cite this article as: Zhou et al:: Algorithm for pre-emptive glycopeptide treatment in patients with haematologic malignancies and an

Enterococcus faecium bloodstream infection. Antimicrobial Resistance and Infection Control 2013 2:24.

\section{Submit your next manuscript to BioMed Central and take full advantage of:}

- Convenient online submission

- Thorough peer review

- No space constraints or color figure charges

- Immediate publication on acceptance

- Inclusion in PubMed, CAS, Scopus and Google Scholar

- Research which is freely available for redistribution 